\title{
Scansion Text Written in English language and Recognized Printed English Character using Bidirectional Associative Memory Network
}

\author{
Aseel W. Ali \\ aseelwaleed@uomosul.edu.iq \\ College of Computer sciences and Mathematics \\ University of Mosul, Mosul, Iraq \\ Received on: 26/06/2011 \\ Accepted on: 03/10/2011
}

\section{ABSTRACT}

The fact that English language is a universal language, so it is necessary to propose a computerized ways to recognize the texts written in English language, which will simplifies the reading of any text, treat it, and deal with it in a least possible time.

The BAM (Bidirectional Associative Memory) network was used to recognize the printed English letters, because it process the small size images of letters in an easy way, also BAM is working in two ways (forward and backward) and store the weights without any amendment, therefore BAM is considered as one of the networks of education controller (Supervised learning).

The recognition of the printed English text was done using the network BAM, while the printed English text was entered to the computer using the scanner, also BAM network used to recognize the letters that have some noise and after training; it gives successful results of recognition about $84.6 \%$.

The aim of this research is to segment and recognize the printed English text, wither it is clear or it have some noise, Matlab R2008a language is used to accomplish this work.

Keywords: Pattern recognitions, Neural Networks, Bidirectional Associative Memory

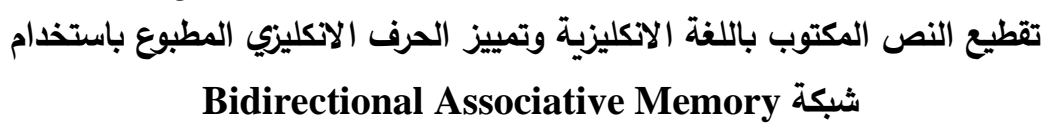

\section{شidirectional Associative Memory شبكة}

$$
\text { كلبة علوم الحاسوب والرياضيات، جامعة الدوصل }
$$

$$
\begin{aligned}
& \text { تاريخ قبول البحث: 2011/10/03 } \\
& \text { تاريخ استلام البحث: 2011/06/26 } \\
& \text { الملخص } \\
& \text { لكون اللغة الانكليزية لغةً عالمية كان لابد من إيجاد طرائق حاسوبية لتمييز النصوص المكتوبة باللغة }
\end{aligned}
$$

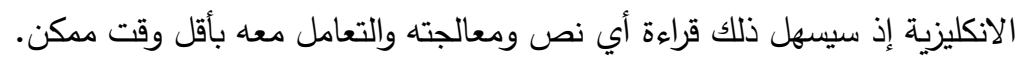

$$
\begin{aligned}
& \text { تم استخدام شبكة Bidirectional Associative Memory) BAM في عملية تمييز الحروف الانكليزية } \\
& \text { المطبوعة لكونها شبكة سهلة التعامل مع الحجم الصغير لصورة الحرف، إذ أنها تعمل باتجاهين (تغذية أمامية وتغذية }
\end{aligned}
$$

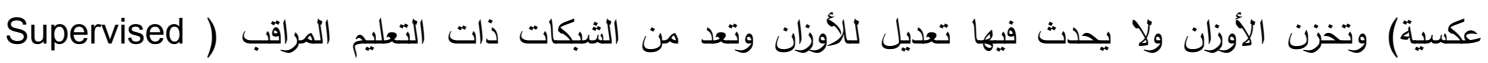

$$
\begin{aligned}
& \text {.(learning } \\
& \text { تم تمييز النص الإنكليزي المطبوع باستخدام شبكة BAM، ولقد تم إدخال النص المكتوب باللغة الانكليزية }
\end{aligned}
$$

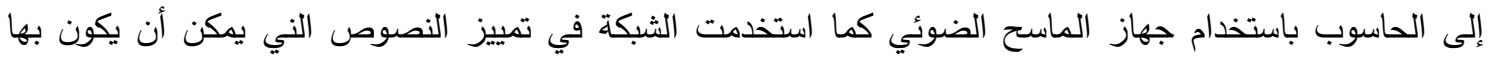

$$
\begin{aligned}
& \text { بعض التشوه وتم التدريب عليها وقد أعطت نتائج ناجحة في عملية التمييز بنسبة 84.6\%. } \\
& \text { الهدف من البحث هو تقطيع وتمييز النصوص الانكليزية المطبوعة سواء كانت واضحة أم مشوهة. } \\
& \text { تم استخدام لغة Matlab R2010a لإنجاز هذا العمل. } \\
& \text { الكلمات المفتاحية: تمييز الانماط، الثبكات العصبية، ذاكرة الارتباط الثنائي هAM. }
\end{aligned}
$$


إن التمييز الضوئي للحروف (Optical Character Recognition) هو تمييز صورة النص المكتوب ميكانيكياً أو الكترونياً، وتكمن أهمية تمييز الرموز والحروف في المجالات التي تستخدم التوثيق الالكتروني إذ يتت تحويل الكتب والوثائق إلى صيغة رقمية، ففي السابق كان يجب كتابة أو نقل هذه الوثائق إلى الحاسبة يدوياً وكانت

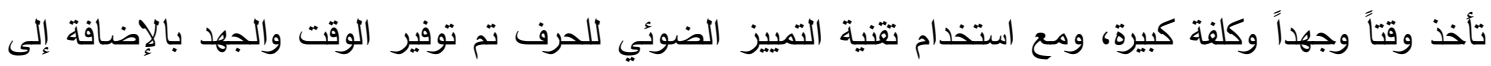
تقليل الكلفة. إن التعرف على الحروف ضوئياً يجعل من الممكن تحرير النص أو البحث عن كلمة ولمة أو جملة وحفظها وتطبيق التقنيات عليها مثل الترجمة الآلية لنص الكلام، الرؤية الحاسوبية، التعرف على على الأنماط، قراءة التصني الثيكات في البنوك، قراءة الجوازات وكذلك في عمليات التوثيق والتي تكون ضرورية في المراكز البحثية والمكتبات الالكترونية ومؤسسات الدولة الأمنية والعامة [9].

2.1 (الدراسات السابقة:

نستعرض في هذه الفقرة الدراسات السابقة التي أنجزت في مجال تمييز الحروف الانكليزية، إذ أن، Anita, 2010 [10 قامت بتصميم نظام حاسوبي لتمييز الحرف الانكليزي باستخدام شبكة البيرسيبترون ذات الطبقة

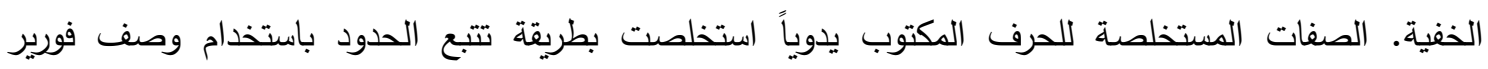
Fourier Descriptor في عام 2008 قام العصبية والمسافات الإقليدية القياسية. في البدء تم تدريب الشبكة العصبية وبعد ذلك استخدمت الثبكة في تمييز الحروف المجهولة والدكتوبة بخط اليد. وفي حالة عدم تطابق الخط اليدوي تستخدم المسافة الاقليدية القياسية لتحسين نسبة التمييز.

في عام 2008 قام Shashank [7] بتمييز الحروف الضوئية OCR وهي احد أول التطبيقات الخاصة بالشبكات العصبية التي حاكت جزئياً التفكير البشري في حقل الذكاء الاصطناعي. وقد تم وصف طريقة عصبية مبسطة لتمييز ومناقثة الحروف الضوئية. في عام 2007 قام الباحث جمال صلاح الدين [12] بإيجاد طريقة لتمييز الحروف الانكليزية المطبوعة باستخدام خوارزميات معينة مثل Boundary algorithm للتقطيع و Following algorithm لاستخلاص الخواص. تم استخدام الماسح الضوئي لغرض سحب النص المطبوع ومن ثم تمييز الحروف. في عام 2002 قامت الباحثة إيمان [11] بالتركيز على أسلوب التمييز القواعدي الذي تقوم فكرته تُشيز الأساسية على استخدام المعلومات التركيبية للأنماط في عملية التصنيف وتمت الاستفادة من خصائصه في تصميم خوارزمية لتمييز بعض الحروف الانكليزية الكبيرة المطبوعة إذ تضمنت عدة مراحل وهي (المعالجة الأولية، استخراج المكونات والعناصر التي مثلت الحروف بالاعتماد على خوارزمية تحويل هاف، تمثيل الحروف بسلسة من بن

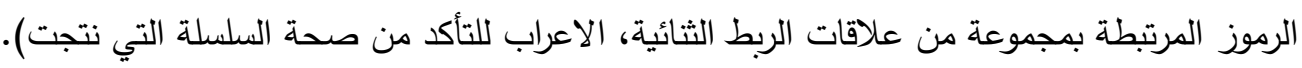

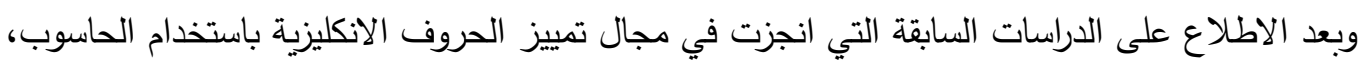
تم تصميم نظام حاسوبي لتمييز الحرف الانكليزي المطبوع باستخدام شبكة BAM لما تملكه من مواصفات كفوهة تسرع في عملية التمييز بالاضافة الى تحقيق نسبة تمييز عالية للحرف الانكليزي. 
2- الثبكة العصبية BAM) Bidirectional Associative Memory):

ان شبكة BAM هي نوع من الثبكات العصبية المتكررة (recurrent neural network) اذ انها قدمت من قبل (Bart kosko 1988) اذ يوجد نوعان من الذاكرة هما Auto associative و

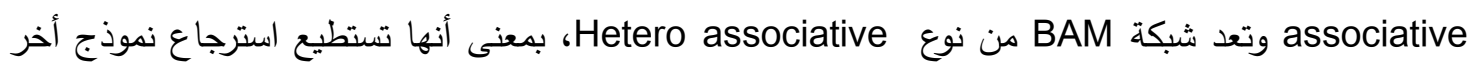

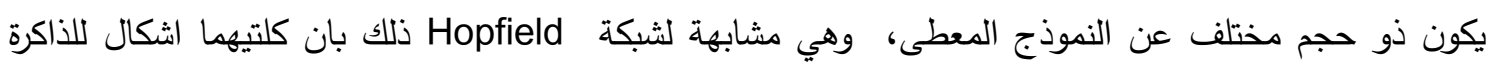
الترابطية الا ان الاخيرة تعيد نموذجا مطابقا بالحجم بينما تعيد BAM أنموذجا مختلفا بالحجم وهي تحتوي على طبقتين [3]. ولثبكة BAM مميزات مهمة منها:

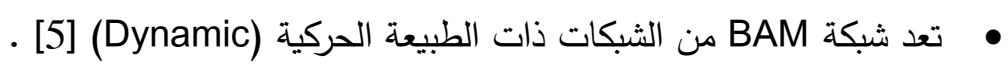

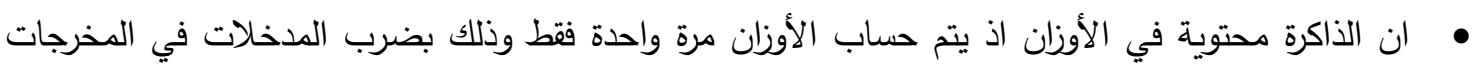

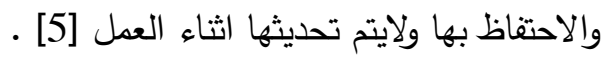
• • • • ان الذاكرة موزعة [4].

كلا المحفزات (النماذج المدخلة) والاستجابة (النماذج الناتجة) هي موجهات عالية جداً (قيمتها كبيرة) [4].

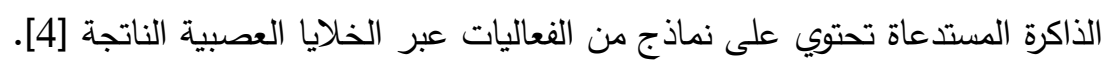

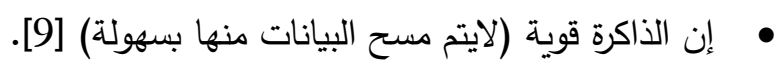

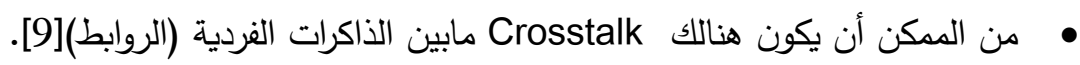

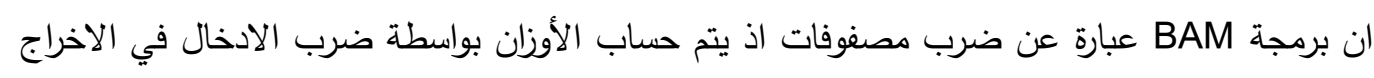
ومن ثم تدريب الثبكة على نماذج لم تكن مدخلة بالضبط [3]. ومن ثم نستخدم الثبكة لتمييز الانماط اذ يكون

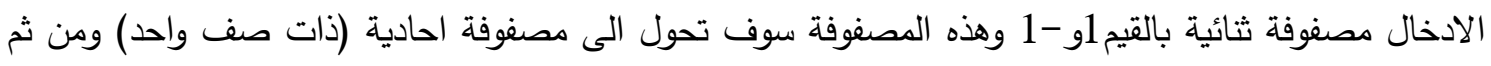
تعطى الى شبكة BAM والتي تحوي على عدد من العصبونات (neurons) مساويا لعدد الادخالات [5][10]

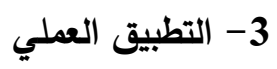
في هذه الفقرة سوف يتم توضيح كيفية تصميم وبناء نظام حاسوبي لتمييز النص الانكليزي المطبوع اذ تم استخدام نظام Matlab 2008 والذي من خلاله يتم عرض النصوص الانكليزية وتمييزها والتعامل معها وقد

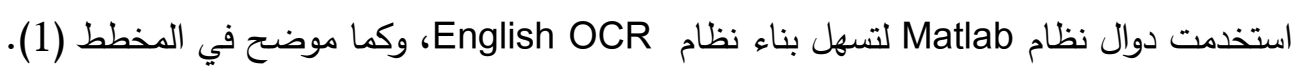




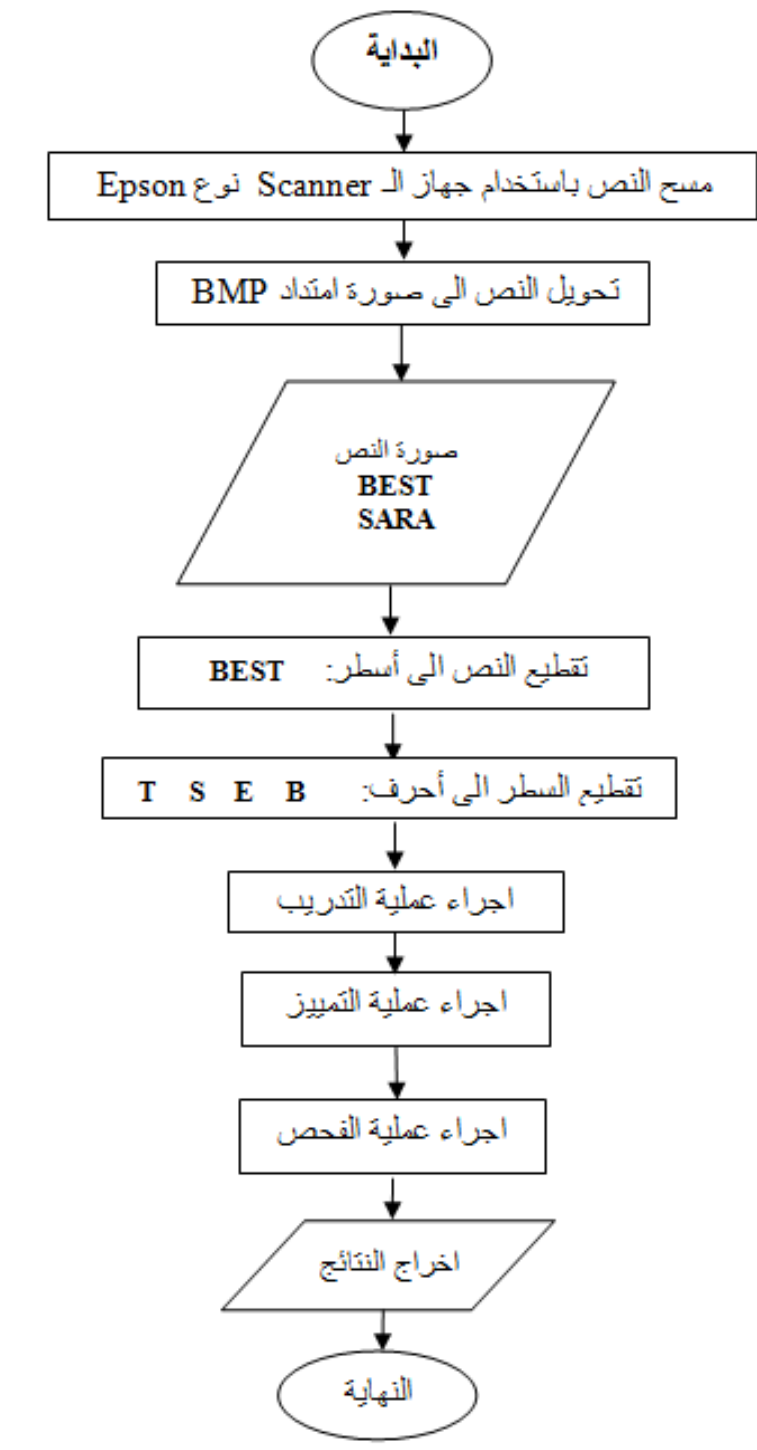

مخطط (1): المخطط الرئيسي لبناء نظام تمبيز الحرف الانكليزي

3-3 مراحل عمل نظام تقطيع وتمييز النص الانكليزي:

1.1.3 مرحلة الحصول على النص: سوف يتم ادخال النص الانكليزي للحصول على معلومات الصورة عن طريق الماسح الضوئي وهذا يمثل المرحلة الاولى في عمل النظام اذ يساعد هذا الجهاز على قراءة نص معين

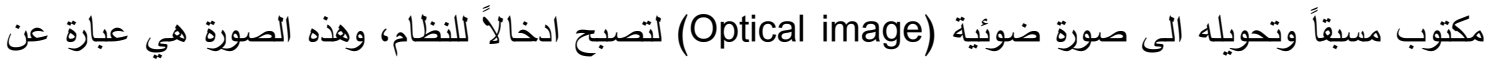

كم هائل من المعلومات يمكن التعامل معها، وكما موضيح بالثكل (1):

ABCDEFGHIJKLMNOPQRSTUVWXYZ
ABCDEFGHIJKLMNOPQRSTUVWXYZ
ABCDEFGHIJKLMNOPQRSTUVWXYZ
SARA
BEST

شكل (1): صورة لنص مسحوب بالماسح الضوئي بأحجام وخطوط مختلفة 
2.1.3 مرحلة التحويل للصورة: اذ يتم تحويل الصورة من صورة رمادية او ملونة الى صورة ثنائية (0،1) وهذا

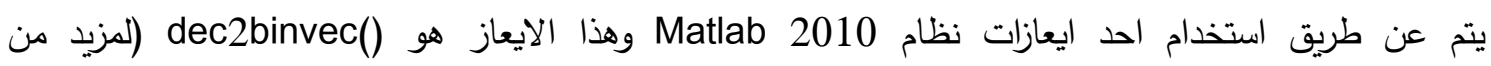
المعلومات انظر التوضيح في الملحق أ).

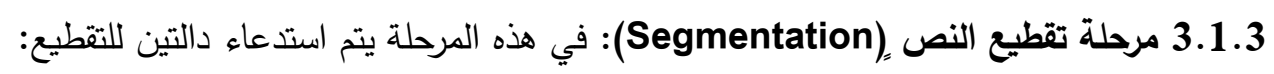

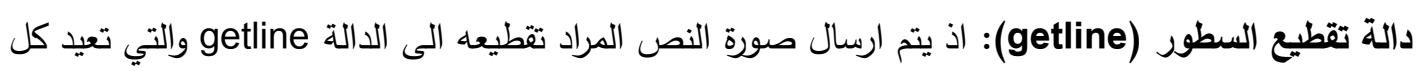
سطر على حدى اذ يتم تتبع الصورة من البداية الى ان يتم تحويلها الى عدد من السطور المستقلة، وكما موضح بالثكل(2) وبالخوارزمية التالية: 1- البداية تتم باعطاء القيم الأولية لدالة تقطيع السطور • بالئكن

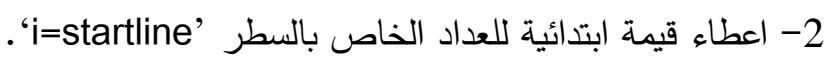

3- اذا تم ايجاد نقطة مضيئة (غير فارغة) فسوف يتم الاحتفاظ بموقعها 'a=i' والانتقال الى الخطوة5.

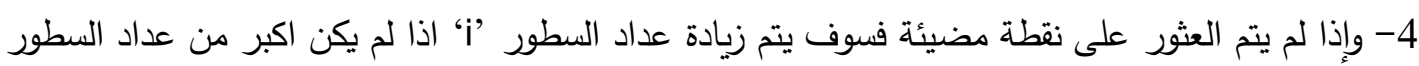
الفعلي للصورة 'row' والعودة الى الخطوة 3. 5- اذا كان الموقع المستحصل عليه 'a' اكبر او مساوياً للقيمة الابتدائية للاسطر 'startline' فسوف يتم الاحتفاظ به ’j=a · · والا فسوف يتم الانتقال الى الخظوة 9. 6- نبحث عن نقطة غير مضيئة (نقطة فارغة) فسوف يتم الاحتفاظ بموقعها 'b=j' والانتقال الى الخطوة 8.

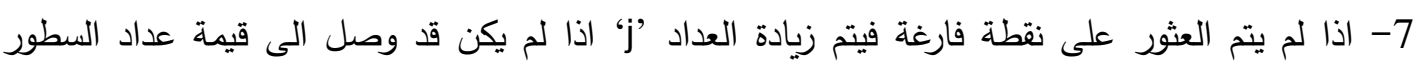

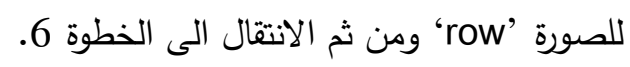

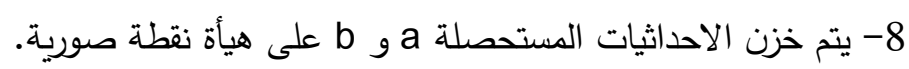

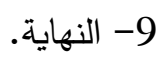

\section{ABCDEFGHIJKLMNOPQRSTUVWXYZ}

$$
\text { شكل (2): تقطيع النص الى اسطر مستقلة }
$$

دالة تقطيع الحروف (getchar): ويأتي عمل الدالة getchar بعد دالة تقطيع السطور اذ يكون السطر هو الأدخال ويكون الاخراج صورة كل حرف بعد تقطيعها الى حروف مستقلة ويتم اخذ السطر وتتبعه افقيا وعموديا الى ان يتم تقطيعه الى حروف مستقلة عن بعضها البعض وكما موضح بالثكل (3) وبالخوارزمية

1- البداية تتم باعطاء القيم الأولية لدالة تقطيع الحروف.

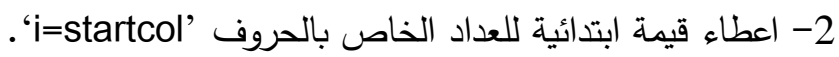

3- اذا تم ايجاد نقطة مضيئة (غير فارغة) فسوف يتم الاحتفاظ بموقعها 'a=i'، والانتقال الى الخطوة 5.

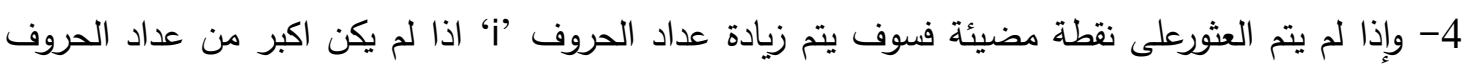

$$
\text { الفعلي للصورة 'col' والعودة الى الخطوة } 3 .
$$

5- اذا كان الموقع المستحصل عليه 'a'، اكبر او مساوياً للقيمة الابتدائية للحروف' 'startcol' فسوف يتم

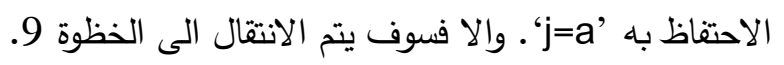

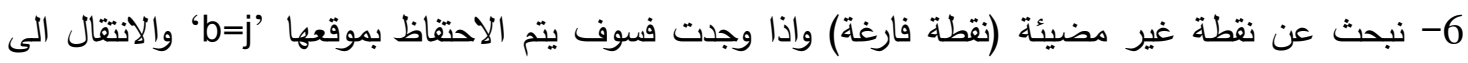


7- اذا لم يتم العثور على نقطة فارغة فيتم زيادة العداد ’j’ اذا لم يكن قد وصل الى قيمة عداد الحروف للصورة

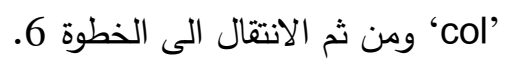

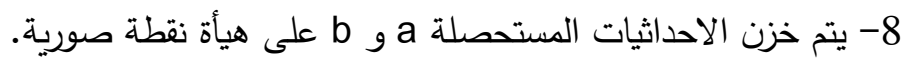

9 - 9 - (النهاية.

\section{A}

شكل (3): أنموذج للحرف المقتطع

4.1.3 مرحلة تحجيم الصورة: وهي تحويل حجم صورة الحرف المستحصل عليه من عملية التقطيع من اي حجم الى الحجم المطلوب التعامل معه وذلك عن طريق استخدام الايعاز :

ch=resizem(ch,[29 26])

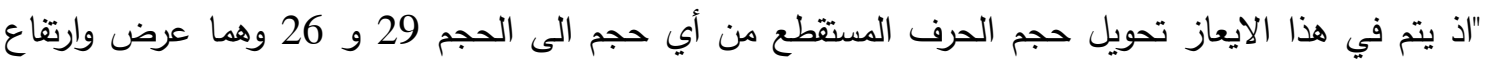
صورة الحرف الواحد الذي تم التعامل معه في هذا البحث" (لمزيد من المعلومات انظر التوضيح في الملحق أ أ). 5.1.3 مرحلة التدربب (Training): في هذه المرحلة يتم تدريب الثبكة على الحروف المراد تمييزها بعد تحديد

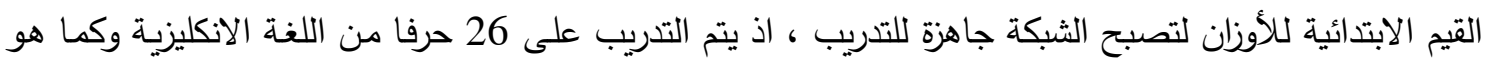

موضح في المخطط (2). 6.1.3 مرحلة التمييز (Recognition): في مرحلة التمييز سوف يتم قراءة الصورة المطلوب اجراء الاختبار

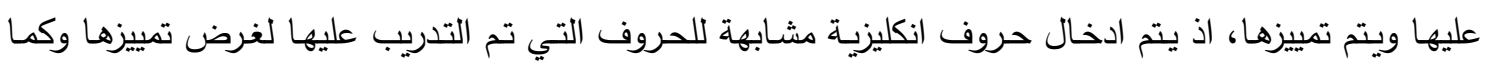

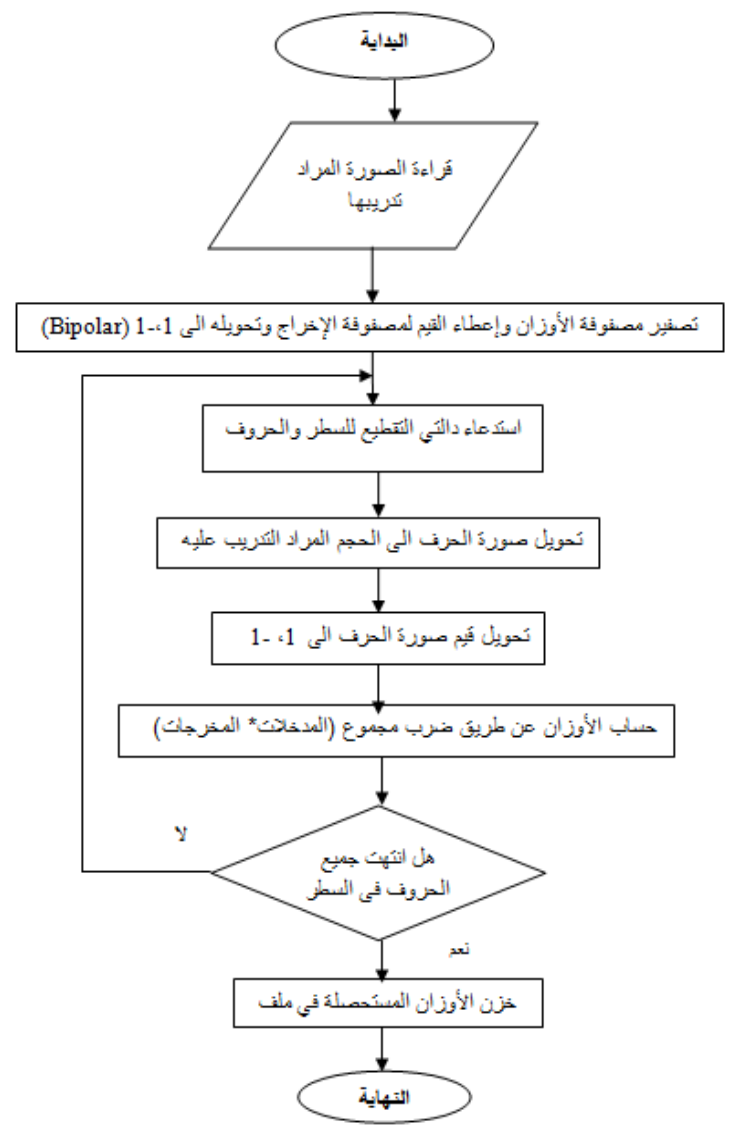

موضح بالمخطط (3).

مخطط (2): تدريب الثبكة العصبية

الاصطناعية BAM 


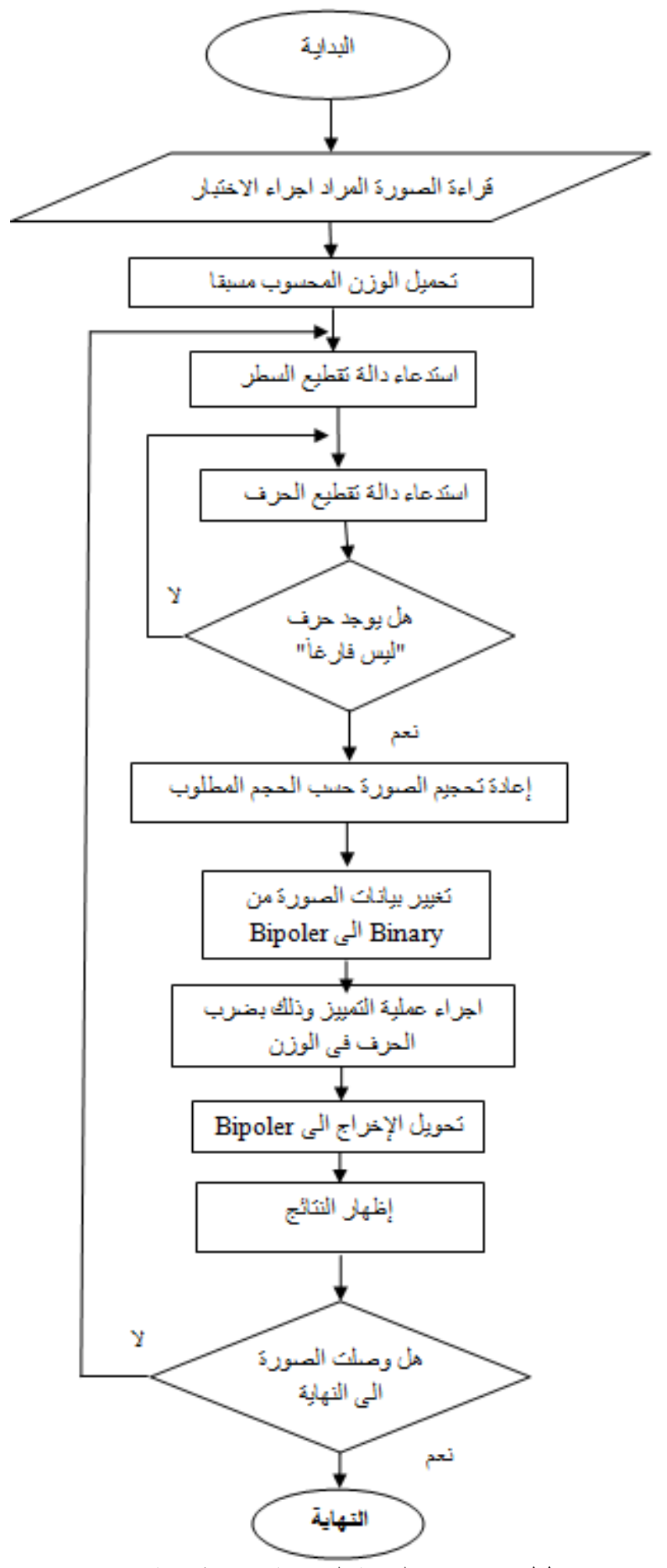

مخطط (3): تمييز الثبكة العصبية الاصطناعية BAM

7.1.3 مرحلة الاختبار (Testing): اذ يتم ادخال أحرف لم يتم التدريب عليها أي انها مختلفة عن الحروف

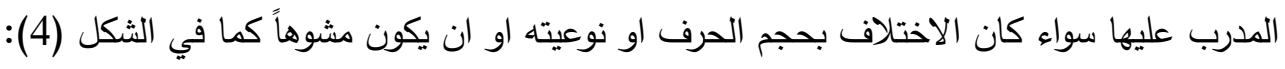

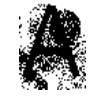

Aكل (4): صورة الحرف المشوه A 
4- نظام تمييز الحروف الانكليزية ضوئياً (English OCR):

عند تنفيذ البرنامج يظهر مايلي:

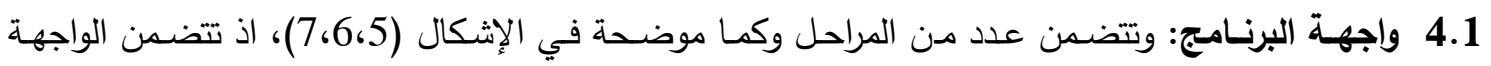
الموضحة في الثكل (5) مائتي:

1. تحميل صورة التدريب: عند الضغط عليها يتم عرض ملفات الصور ثم يتم اختيار واحدة من الصور المراد

$$
\text { التدريب عليها. }
$$

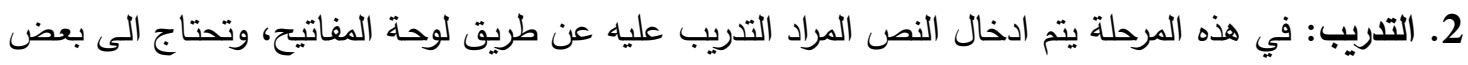

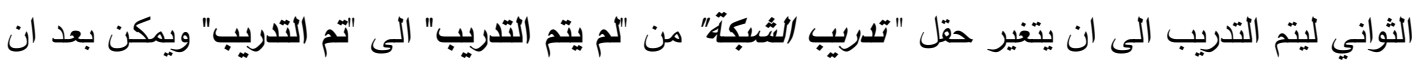

يتم التدريب بثكل كامل ان نضغط على خانة "عرض مصفوفة الأوزان" لرؤية مصفوفة الأوزان.

3. تحميل الصورة المراد تمييزها:عند الضغط على هذه الخانة يتم عرض عدد من الصور للنصوص المراد

$$
\text { تمييزها فيتم اختيار احداها. }
$$

4. تمييز: عند الضغط عليها يتم تمييز النص كاملا وعرضه بشكل سطر واحدا. واهد . 5. صورة الحرف: في هذه المرحلة يتم تقطيع السطر الى احرف مستقلة وعرض كل حرف على حدة. 6. ناتج عملية التمييز: في هذه المرحلة يتم عرض نتائج عملية التمييز كاملاً.

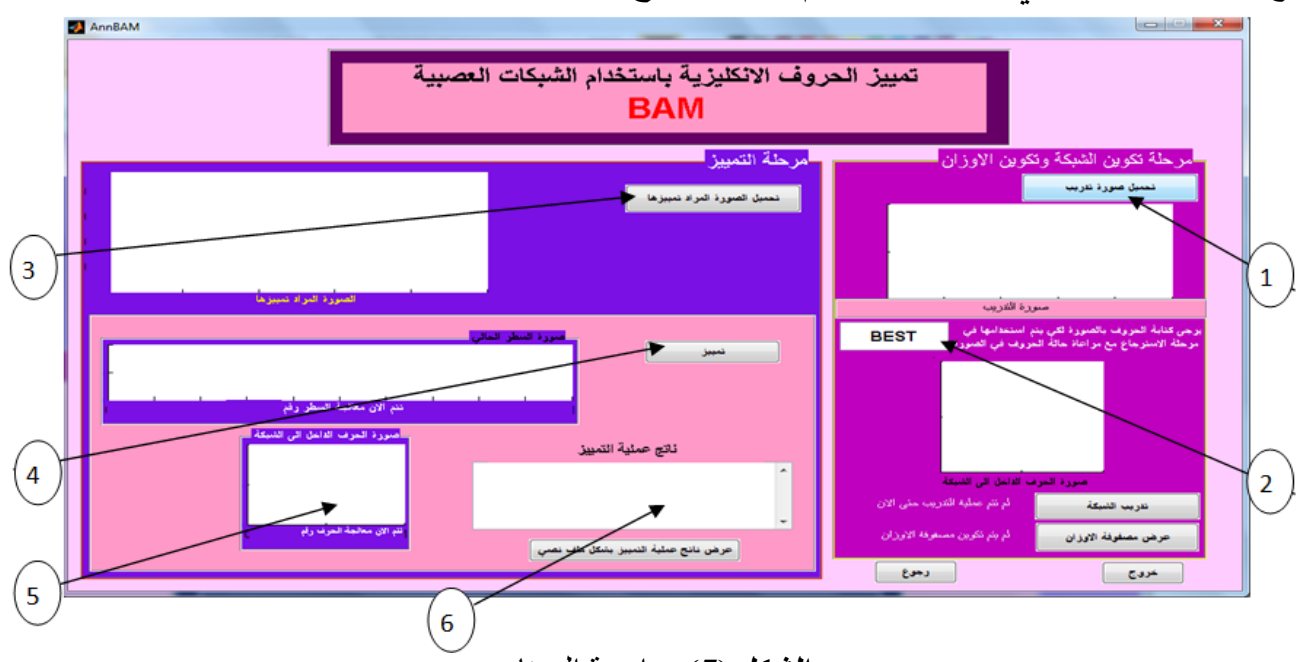

يوضح الثكل (6) محتويات الحقول كافة في الواجهل (5): الزُريسية البرنامجنتطبيق العملي لهذا البحث.

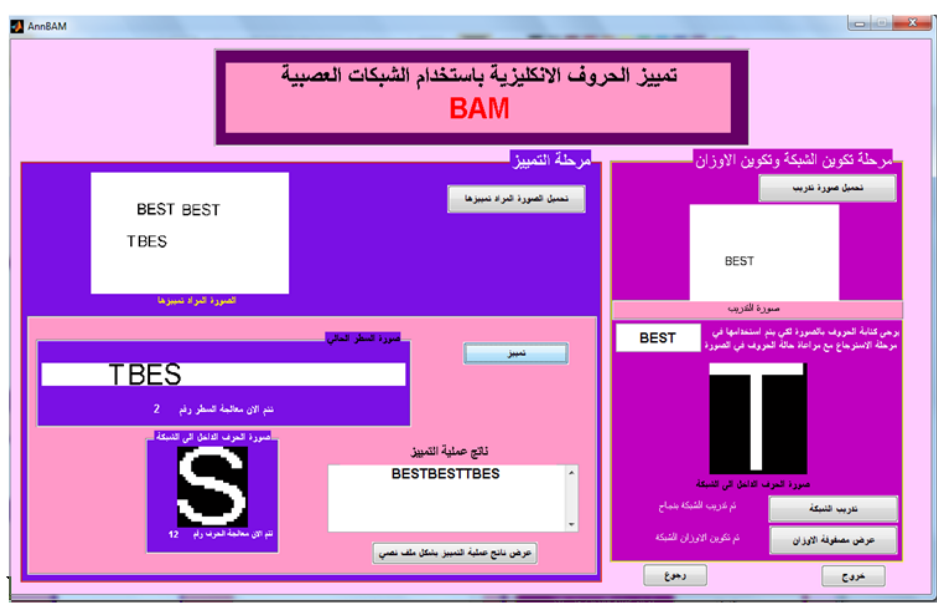

الثكل (6): مثال لصورة تحتوي على الحروف المطلوب تدريب الثبكة عليها 
وعند الضغط على "عرض الأوزلن" تظهر مصفوفة الأوزان كما في الثكل (7):

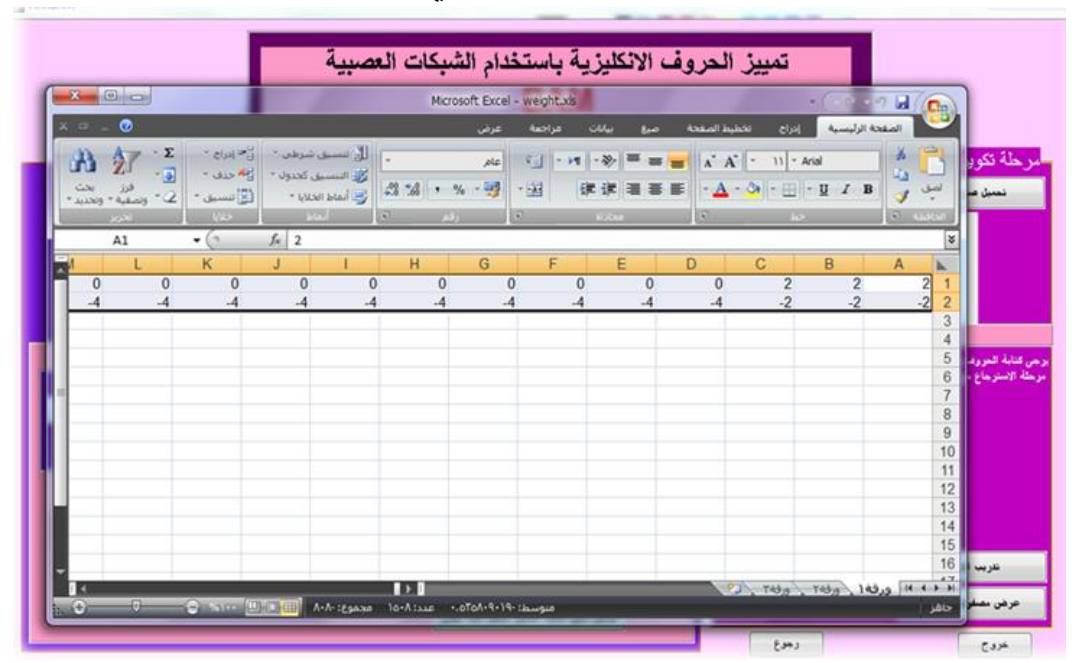

الثكل (7): تدريب الثبكة على مصفوفة الأوزان

:Testing 2-4 الاختبار 2-4

وتتضمن هذه المرحلة ادخال اكثر من صورة ومقارنة الناتج مع مرحلة التدريب وعند النقر على خانة

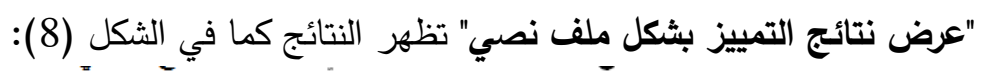

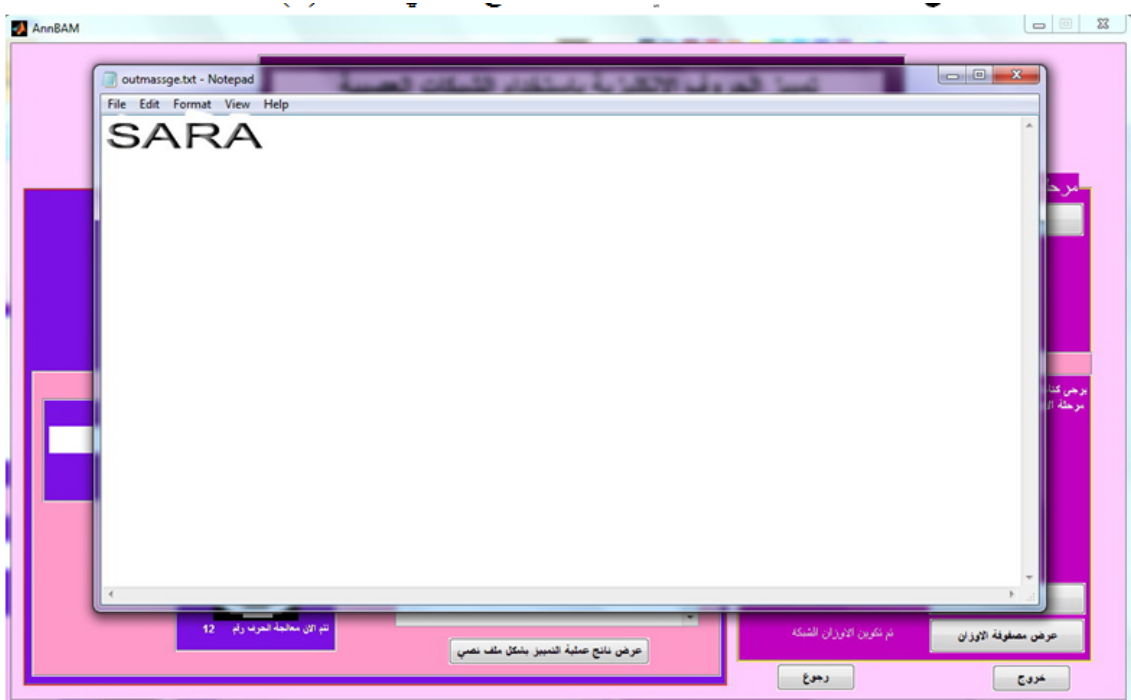

الشكل (8): عرض النتائج بشكل ملف نصي

ويكون الاخراج النهائي باعطاء كل حرف رقماً مكتوباً بشكل (1 و Bipolar) اذ ان ان الحرف كلما ورد يأخذ نفس

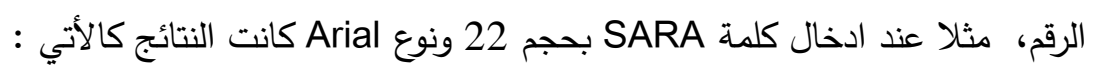

SARA

$\begin{array}{lrr}\mathrm{S} & -1 & -1 \\ \mathrm{~A} & -1 & 1 \\ \mathrm{R} & 1 & -1 \\ \mathrm{~A} & -1 & 1\end{array}$


وعند ادخال نفس الكلمة بنفس الحجم والنوع ولكن بشكل Bold كانت النتائج مطابقة للنتائج السابقة وكما موضح ادناه:

\section{SARA}
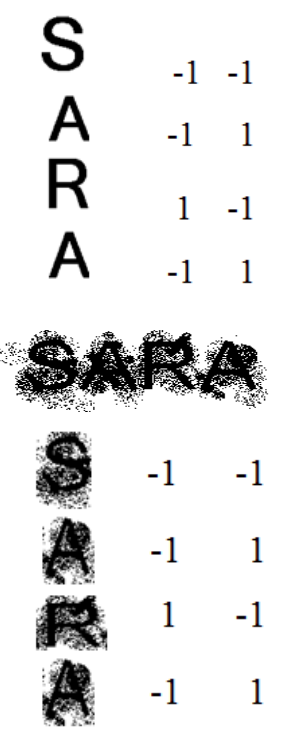

$$
\text { وعند تثويه النص سوف يعطي نفس الناتج و كالاتي: }
$$

وعند ادخال الكلمة BEST بنفس الحجم والنوع كانت النتائج كما موضح ادناه:

\section{BEST}

$$
\begin{array}{ccc}
\mathbf{B} & -1 & -1 \\
\mathbf{E} & -1 & 1 \\
\mathbf{S} & 1 & -1 \\
\mathrm{~T} & 1 & 1
\end{array}
$$

\section{BEST}

إدخال الصورة نفسها بخط مختلف وتعطي نفس الناتج :

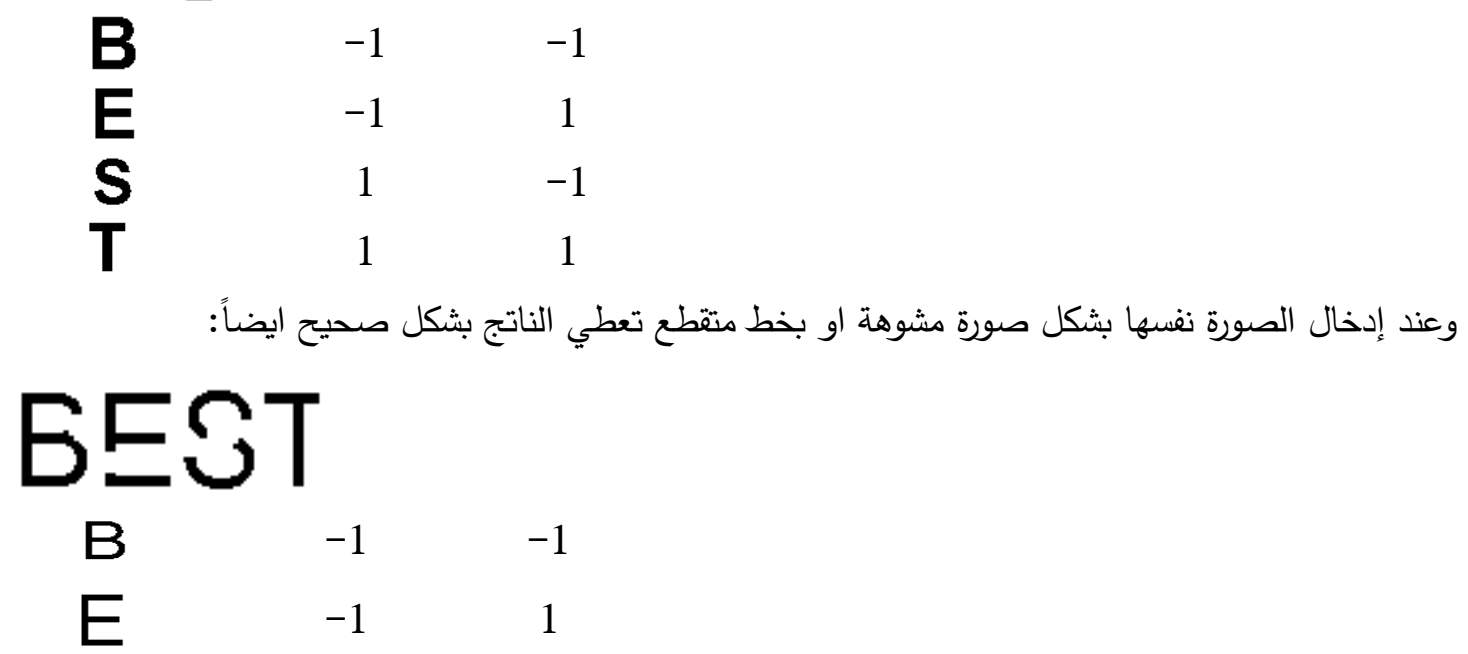




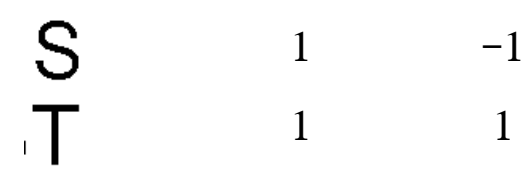

5- النتائج والمناقثة:

تم تدريب النظام على 26 حرفاً انكليزياً مطبوعاً و20 نصاً انكليزياً مطبوعاً ومسحوباً بجهاز الماسح الضوئي وكانت عملية التمييز في مرحلة التدريب قد حققت نجاحاً بنسبة 99\% . ولاختبار عملية التميييز تم اختبار النظام على 10 نصوص انكليزية وحقت نسبة نجاح قدرها 80\% كما ان نسبة التمييز للحرف في مرحلة الاختبار حقت نسبة نجاح وقدرها 84.615\% وقد تم اعتماد القانون ادناه في عملية حساب نسب النجاح:

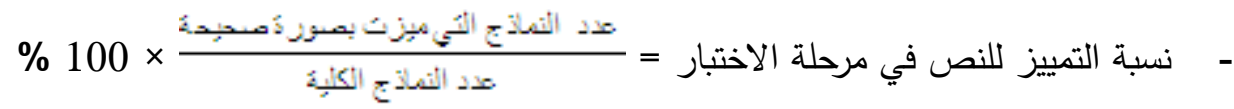
"اذ أن كل نموذج يقصد به كلمة معينة أي

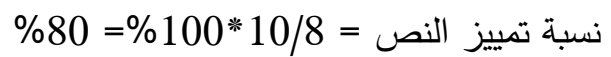

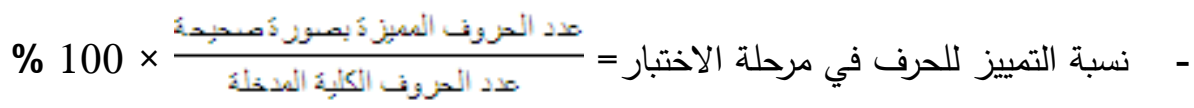
ن84.615=100*26/22 = نسبة تمييز الحروف

5- الاستنتاجات:

• من خلال هذا البحث نستتتج ان شبكة BAM من الثبكات الملائمة لتمييز الأنماط والنصوص اذ تكون سهلة

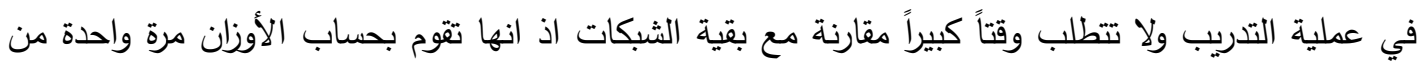

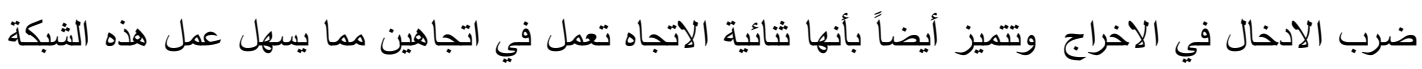
عند استخدامها في تمييز الأنماط والنصوص. • ان شبكة BAM تستخدم لتمييز نماذج الحروف الانكليزية (A-Z). وبما ان معمارية الشبكة المثالية القريبة لهذه الخوارزمية قد وجدت، لذلك فمن الممكن إن نصنف بشكل صحيح او نميز النموذج المخزون.

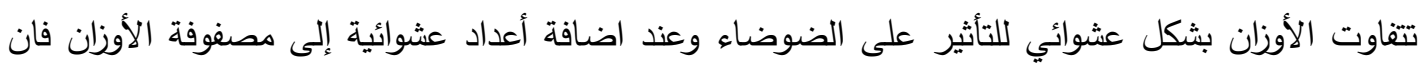

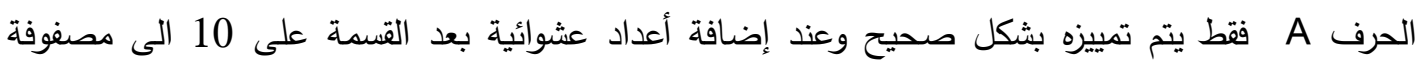

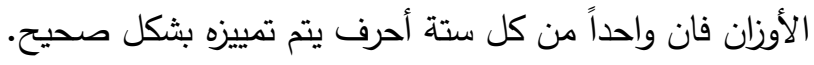

ه. نوصي من خلال بحثا ان يكون التمييز في مجالات شتى لمختلف التطبيقات التي يمكن ان تستعمل في

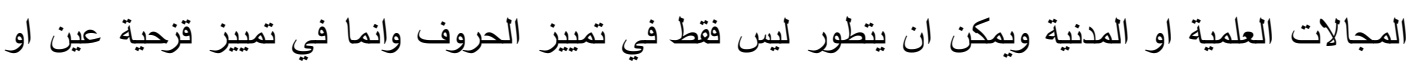
بصمة الابهام او تمييز التواقيع لكي تستخدم في الدوائر الحكومية المختلفة المنتشرة.

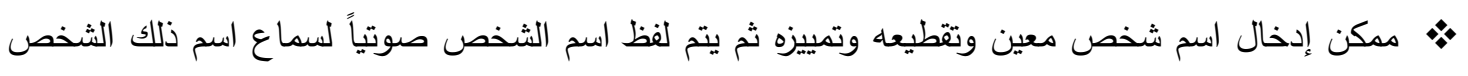
وهذا مطبق فعليا في اجهز الموبايل الحديثة ولكن نوصي بتجربة هذا العمل مختبريا ليتم الاستفادة منه في لئي

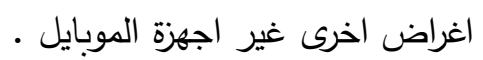




\section{إدمادر}

[1] Anita Pal1 \& Dayashankar Singh, Handwritten English Character Recognition Using Neural Network, International Journal of Computer Science \& CommunicationVol. 1, No. 2, July-December 2010, pp. 141-144.

[2] B.kosko, "Adaptive bidirectional associative memories" applied optics, vol 26, no.23, pp.4947-4960, 1987.

[3] Bart Kosko, Bidirectional associative memories, University of southern California , Losangeles, published in jornall IEEE, volume 18, pp.42-60, 1988.

[4] http :/ ar . wikibookes . org / wiki Artificial neural net works.

[5] Michael Negnevitsky, "Artificial Intelligence , A Guide to Intelligent Systems", second edition, 2007.

[6] Richard Duda and Peter Hart et. al. Pattern classification $2^{\text {nd }}$ edition, December 28, 2006.

[7] Shashank Araokar,Visual Character Recognition using Artificial Neural Networks, MGM's College of Engineering and Technology,University of Mumbai, India,2008.

[8] Sumit Saha, Handwritten Character Recognition Using Neural Network and Euclidean Distance Metric, Assam University - Department of Mathematics, April $14,2008$.

[9] www.alepposoft .com / Artificial neural net works.

[10] Xin-geliu,raphr.martin,min w4,and mei-lantang," global Exponential stability of bidirectional associative memory neural networkes with time delay " , 2008 , IEEE .

$$
\begin{aligned}
& \text { [11] العبيدي ايمان ، تمييز الحروف الانكليزية المطبوعة باستخدام اسلوب التمييز القواعدي، بحث مقدم لنيل }
\end{aligned}
$$

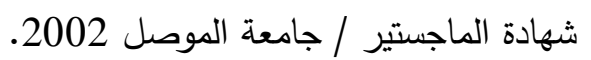

$$
\begin{aligned}
& \text { [12 النعيمي جمال ، تمييز الحرف الانكليزي المطبوع، بحث منشور في المجلة العراقية للعلوم الاحصائية، } \\
& \text { مجلد (5)، العدد(8)، ص 166 160-180، } 2005 \text { ـ }
\end{aligned}
$$




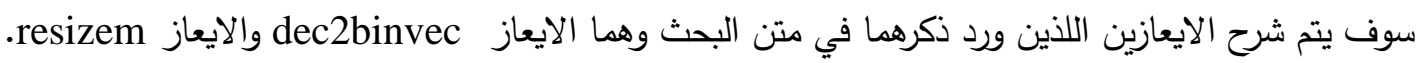

\section{dec2binvec}

Convert digital input and output decimal value to binary vector.

out $=$ dec2binvec $(\operatorname{dec})$

\section{Syntax}

$$
\text { يحول الادخال الرقمي والاخراج العشري الى متجه ثنائي. }
$$

out $=$ dec2binvec $($ dec, bits $)$

\section{Arguments}

Dec A decimal value. dec must be non-negative.

bits Number of bits used to represent the decimal number.

Out A logical array containing the binary vector.

\section{Examples}

To convert the decimal value 23 to a binvec value:

dec2binvec $(23)$

ans $=\begin{array}{lllll}1 & 1 & 1 & 0 & 1\end{array}$

To convert the decimal value 23 to a binvec value using six bits:

dec2binvec $(23,6)$

ans $=\begin{array}{llllll}1 & 1 & 1 & 0 & 1 & 0\end{array}$

To convert the decimal value 23 to a binvec value using four bits, then the result uses

five bits. This is the minimum number of bits required to represent the number.

dec2binvec $(23,4)$

ans $=\begin{array}{lllll}1 & 1 & 1 & 0 & 1\end{array}$

\section{resizem}

Resize regular data grid.

اعادة تحجيم شبكة البيانات النظامية.

$\mathrm{Z}=\operatorname{resizem}(\mathrm{Z} 1$, scale $)$

\section{Syntax}

$\mathrm{Z}=\operatorname{resizem}(\mathrm{Z} 1$, [numrows numcols $])$

$[Z, R]=\operatorname{resizem}(Z 1$, scale, $R 1)$

$[\mathrm{Z}, \mathrm{R}]=\operatorname{resizem}(\mathrm{Z} 1$, [numrows numcols $], \mathrm{R} 1)$

$[\ldots]=\operatorname{resizem}(\ldots$, method $)$

\section{Example}

Double the size of a grid then reduce it using different methods:

$\mathrm{Z}=[12 ; 34]$

$\mathrm{Z}=$

12

34

neargrid $=\operatorname{resizem}(\mathrm{Z}, 2)$

neargrid $=\begin{array}{llll}1 & 1 & 2 & 2\end{array}$

$\begin{array}{llll}1 & 1 & 2 & 2\end{array}$

$\begin{array}{llll}3 & 3 & 4 & 4\end{array}$

3344 
bilingrid $=$ resizem $(\mathrm{Z}, 2$, 'bilinear')

bilingrid $=\begin{array}{llll}1.0000 & 1.3333 & 1.6667 & 2.0000\end{array}$

$\begin{array}{llll}1.6667 & 2.0000 & 2.3333 & 2.6667\end{array}$

$\begin{array}{llll}2.3333 & 2.6667 & 3.0000 & 3.3333\end{array}$

$\begin{array}{llll}3.0000 & 3.3333 & 3.6667 & 4.0000\end{array}$

bicubgrid $=$ resizem $($ bilingrid, $[32$ 2],'bicubic')

bicubgrid $=0.7406 \quad 1.2994$

$1.6616 \quad 2.3462$

$1.9718 \quad 2.5306$ 